Article

\title{
An Automatic Measurement Method for Absolute Depth of Objects in Two Monocular Images Based on SIFT Feature
}

\author{
Lixin He ${ }^{1,2,3, *}$, Jing Yang ${ }^{2, *}$, Bin Kong ${ }^{2}$ and Can Wang ${ }^{2}$ \\ 1 Department of Automation, University of Science and Technology of China, Hefei 230027, China \\ 2 Hefei Institute of Intelligent Machines, Chinese Academy of Sciences, Hefei 230031, China; \\ bkong@iim.ac.cn (B.K.); cwang@iim.ac.cn (C.W.) \\ 3 The key lab of Network and Intelligent Information Processing, Hefei University, Hefei 230601, China \\ * Correspondence: hlxiniim@mail.ustc.edu.cn (L.H.); Tel.: +86-138-0569-1938 (L.H.); \\ jyang@iim.ac.cn (J.Y.); Tel.: +86-139-5510-7206 (J.Y.)
}

\begin{abstract}
It is one of very important and basic problem in compute vision field that recovering depth information of objects from two-dimensional images. In view of the shortcomings of existing methods of depth estimation, a novel approach based on SIFT (the Scale Invariant Feature Transform) is presented in this paper. The approach can estimate the depths of objects in two images which are captured by an un-calibrated ordinary monocular camera. In this approach, above all, the first image is captured. All of the camera parameters remain unchanged, and the second image is acquired after moving the camera a distance $d$ along the optical axis. Then image segmentation and SIFT feature extraction are implemented on the two images separately, and objects in the images are matched. Lastly, an object depth can be computed by the lengths of a pair of straight line segments. In order to ensure that the best appropriate a pair of straight line segments are chose and reduce the computation, the theory of convex hull and the knowledge of triangle similarity are employed. The experimental results show our approach is effective and practical.
\end{abstract}

Keywords: monocular image; image segment; SIFT; depth measurement; convex hull

\section{Introduction}

Acquiring depth information from 2D images is one of fundamental problems in machine vision, and it can be applied to many fields such as restoration of 3D scene, planning of robot walking route, et.al. Especially, the depths of interesting objects in images are very useful, for instance, the distances from obstacles in the road, frontal vehicles, and the traffic light must be knew when an unmanned vehicle is running on the road. The depth information also can be used to pattern recognition[1,2].

At present, the methods of obtaining the depth information of objects from 2D image are mainly two major categories: the stereo vision based on binocular(or multi-nocular)[3-5], and the stereo vision based on monocular. At least, the two camera devices must be provided in the first class method. The every camera intrinsic parameters and the parameters of spatial relationship between any two cameras are also provided. It is means the camera calibration is need in the first class method. Bumblebee is a kind of product of stereo vision based on binocular, and a light-field camera also can get the depth information for its micro-lens array[6-10]. But both of them are expensive.

The second class method includes Depth From Focus(DFF) and Depth From Defocus(DFD). In the DFF method[11,12], a lot of images on the same scene with different camera optical parameters should be took. Then a full focus image is formed by using the pixels which is in focus and in the images. At last, the depth map can be got by analyzing every pixel in the full focus image and the 
camera parameters when the pixel is get. Only one camera is need in this method, but its application is very limited for a large number of images of the same scene must be got.

The DFD was first proposed by Pentland[13] in 1987, and was improved by Subbarao[14] and Rajagopalan[15]. In 2008, defocus was modeled as an anisotropic thermal diffusion process by Favaro[16] et al, and this improvement has a better result. But two or more images which are took in different camera intrinsic should be supplied in the above improve method. Zhou and Sim[17] presented an original approach that depth map could be estimated from a single defocus image. In this method, firstly, the defocus image is re-blurred using a known Guassian kernel. Then the depth information at edge in image can be obtained by the ratio between the gradient of the defocus image and the re-blurred one. Lastly, the depth at edge locations is propagated to the entire image by solving the optimization problem. All above approach based on defocus could not tell the reason of blur edge in image: it is caused by either blur texture ambiguity or the focal plane ambiguity. And it could not get the absolute depth information unless the camera was calibrated.

The coded aperture method proposed by Zhou[18] et al. It could estimate a better depth map, but the shape of camera aperture must be modified. Kouskouridas[19] et al. used SIFT to acquire the absolute depth of the objects in image. In this method, a database must be built firstly. The database contains 5 or more images which are took from different object distance or angle of view to every object measured. The object distances are acquired by laser device and stored at the database. The data in the database will be used to train the algorithm. It could not estimate the depth of object if the object is not in the database. This means it only can compute the absolute depth of object that has stored in the database. This approach has a complex operation step and a limited application scope. Fang[20] employed a structure forest framework to extract the depth information from a single color image. The method can achieve quasi real-time performance, but the accuracy needs to be improved, especially to indoor scene.

In this paper, we proposed a novel approach to compute the absolute depth of target object (It is given beforehand that which objects are target objects). It only needs a monocular camera and no calibrating. Firstly, the image $A$ was captured, and then the image $B$ is captured after moving the camera a distance $d$ along the optical axis. The camera intrinsic parameters are kept constant during the whole process. Secondly, we get the objects by segmenting the two images, respectively. Simultaneously, the SIFT points are detected in the two images, respectively. And the image of same object in the two images should be matched after completing the SIFT points matched. At last, the absolute depth of object can be computed by using the corresponding the lenths of two straight line segments in the two images. The first straight line segment is composed of two SIFT feature points in image $A$, and the second one is composed of two corresponding matching SIFT feature points in image $B$.

\section{The Basic Principles and Algorithm Steps}

The basic principles of imaging can be modeled as

$$
\frac{1}{f}=\frac{1}{u}+\frac{1}{v}
$$

Where $f$ is the focal length. $u$ is object distance(namely depth), and $v$ is the distance between the image plane and lens.

As show in Figure 1, we assume that the object distance is $u$ and the high of image of object is $h_{1}$ in the first photograph. In the second one, they are $u+d$ and $h_{2}$, respectively. Because the camera intrinsic is unchanged during the two times photograph, the relation of $h_{1}$ and $h_{2}$ can be formulated as

$$
h_{2}=k h_{1}
$$

where $k>0$ and $k \in R$, and $R$ is the set of real number. 


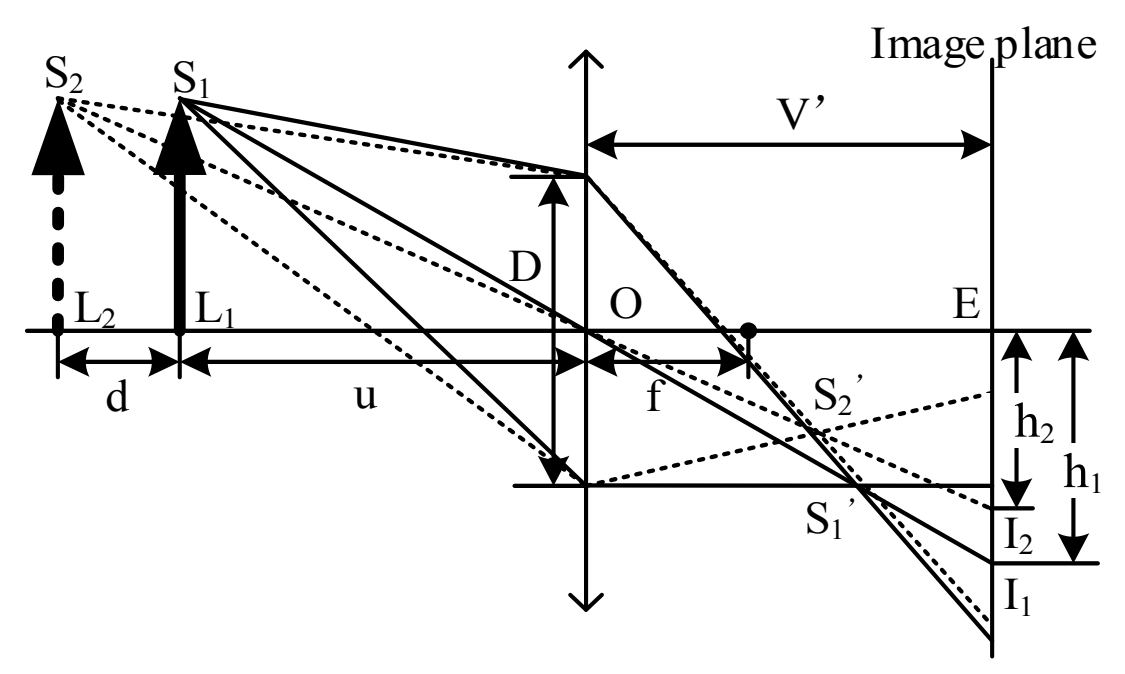

Figure 1. Two times imaging of lens. $D$ is the diameter of the camera aperture. $V^{\prime}$ is the distance between the camera lens and the image plane. $S_{1}$ and $S_{2}$ are the same point $S$ on the imaging object $S L$, In the first imaging, the object distance is $u$, and the imaging object $S L$ is located at $S_{1} L_{1}$, and the $S_{1}{ }^{\prime}$ is the focal point of $S$. In the second imaging, the object distance is $u+d$, and the imaging object $S L$ is located at $S_{2} L_{2}$, and the $S_{2}{ }^{\prime}$ is the focal point of $S$.

According to the basic principles of imaging and the knowledge of triangle, the object distance $u$ can be computed by the following formulation.

$$
u=\frac{h_{2}}{h_{1}-h_{2}} d
$$

Usually, $h_{1}$ and $h_{2}$ is the high of image of object. At fact, they can be the distance of two feature points on an object, too. Therefore, we can compute the depth $u$ if we detect two pair of correspond feature points of the same object in two images.

Figure 2 shows the overview of our method of estimating the object depth. It mainly includes 5 steps as follows:

Step 1: Holding the camera intrinsic constant, we take the image $A$ and $B$ when the object distance is $u$ and $u+d$, respectively.

Step 2: Images of objects (namely sub-regions) are obtained by segmenting the image $A$ and $B$, respectively. Meanwhile, we detect the SIFT feature points in the image $A$ and $B$, then, match the points.

Step 3: Using the result of segmentation and match of feature points, we can match the images of objects.

Step 4: A pair of straight line segments are chosen from the image $A$ and $B$. During the process, the theory of the convex hull is used to decrease the computation complexity, and the knowledge of similarity triangle is used to avoid the wrong straight line straight are chose. The lengths of the pair of straight line segments will be used to compute the depth of object.

Step 5: The depth of object can be computed by the length of the pair of straight line segments. 


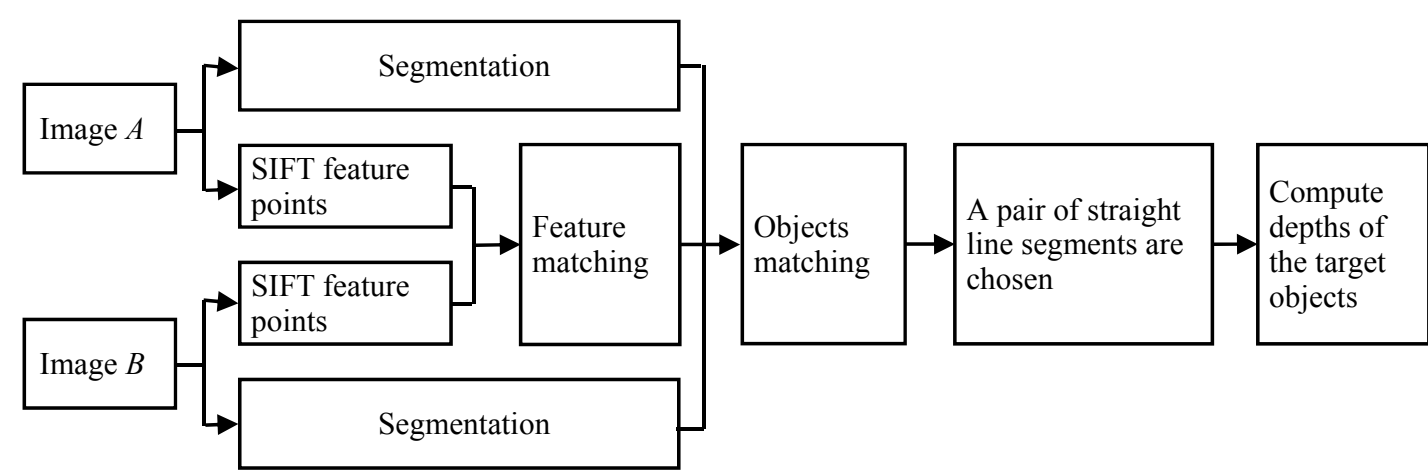

$\begin{array}{lllll}\text { Step } 1 & \text { Step } 2 & \text { Step } 3 & \text { Step } 4 & \text { Step } 5\end{array}$

Figure 2. The overview of our depth measurement method.

\section{Matching the Images of Objects}

Matching the images of objects could not be doing unless both the segmentation and detecting SIFT feature points are completed. At present, there are a number of methods of segmentation. The LBF(Local Binary Fitting Energy) method[21,22] is employed in this paper , because it have a better segmentation result, especially to the images with intensity inhomogeneity. The SIFT feature points are invariant to image scale and rotation, and robust matching across a substantial range of affine distortion, addition of noise, and change in illumination[23,24].

We assume that the image $A$ is partitioned into $m$ subregions by the LBF image segmentation approach. The $m$ subregions are $A_{1}, A_{2}, \ldots, A_{m}$, and $X_{i}$ is the SIFT feature points set of the $A_{i}$ subregion. where $i=1,2, \ldots, m$. Similarly, the image $B$ is segmented into $n$ subregions: $B_{1}, B_{2}, \ldots, B_{n}$, and $Y_{j}$ is the SIFT feature points set of $B_{j}$. where $j=1,2, \ldots, n$. $Z i$ is the matching feature points set of $X_{i}$. Therefore, $Z_{i} \subseteq \quad\left(Y_{1} \cup Y_{2} \cup \ldots \cup Y_{n}\right)$. The $\operatorname{card}(S)$ means the number of elements in the set $S$.

$$
\begin{aligned}
& Y_{k}=\underset{Y_{j}}{\arg \max }\left(\operatorname{card}\left(Z_{i} \cap Y_{j}\right)\right) \\
& \frac{\operatorname{card}\left(Y_{\mathrm{k}}\right)}{\operatorname{card}\left(X_{\mathrm{i}}\right)}>T_{p}
\end{aligned}
$$

where $T_{p}$ is a threshold. The object $A_{i}$ is matched with $B_{k}$ if both the formula (4) and (5) are satisfied, namely, the subregion $A_{i}$ in image $A$ and the subregion $B_{k}$ in image $B$ are the images of the same object.

\section{Selecting the Straight Line Segments}

\subsection{Theoretical Error Analysis}

We can compute the depth of objects using the formula (3) if the length of the two straight line segments is known. In theory, the first straight line segment consist of any two SIFT feature points of an object in image $A$, and the second one consist of the two corresponding matching SIFT feature points in image $B$. There is always a certain error in the matching SIFT points for the Nearest Neighbor Distance method is adopted to match the points. For example, we assume that the matching point of the SIFT feature point $P_{a}$ in image $A$ is the $P_{b}$ in image $B$ theoretically, but we get the matching point $P_{b}{ }^{\prime}$ at fact by the method. The distance between $P_{b}$ and $P_{b}{ }^{\prime}$ is the error. Although, 
the error is very little, even no more than one pixel, the accuracy of estimating depth of object is depended on it.

We assume the length of the straight line segment which consist of two SIFT feature points on an object in image $A$ is $L_{1}$, and the length of the matching line segment is $L_{2}$ in image $B$, theoretically. But we obtain the length is $L_{2}{ }^{\prime}$ in fact for there are matching point error. The difference between $L_{2}$ and $L_{2}{ }^{\prime}$ is $\Delta=L_{2} 2^{\prime}-L_{2}$, and the relation of two length is $L_{2}=k L_{1}$. We assume that $U$ is ground truth of depth of object, and $U^{\prime}$ is the computed depth of object by the formula (3), and we have

$$
\begin{aligned}
& U=\frac{L_{2}}{L_{1}-L_{2}} d \\
& U^{\prime}=\frac{L_{2}{ }^{\prime}}{L_{1}-L_{2}{ }^{\prime}} d=\frac{L_{2}+\Delta}{L_{1}-L_{2}-\Delta} d \\
& e=\left|\frac{U^{\prime}-U}{U}\right|=\left|\frac{\frac{\Delta}{1-k}}{L_{1}-\frac{\Delta}{1-k}}\right| \frac{1}{k}=\left|\frac{J}{L_{1}-J}\right| \frac{1}{k}
\end{aligned}
$$

Where $e$ is percentage error of depth measurement, $J=\frac{\Delta}{1-k}$.

Normally, the $\Delta$ is very little, no more than several pixels, even a single pixel, because $\Delta$ is produced by the matching error of SIFT feature points. We assume $|\Delta| \leqslant 3$ pixels, and $0.75 \leqslant$ $k \leqslant 0.9$, then $0 \leqslant|j| \leqslant 30$. The Figure 3 shows the relations of $e$ to $k$ and $\Delta$. Figure 3(a) shows the error curve when $\Delta<=0$. Figure 3(b) shows the error curve when $\Delta>0$. The range of $e$ value was so large that the curve is hard to see when the $L_{1}$ more than 50 in Figure 3(b), therefore, it was divided into two parts, as the Figure 3(c) and (d). From the Figure 3 and formula (8), we know: (1) that the $e$ increase rapidly when $L_{1} \rightarrow J$, (2)and that the $L_{1}$ is longer, the $e$ is smaller, when the value of $L_{1}$ is larger than a certain value (e.g. 50) and the variable $\Delta$ and $k$ is unchanged. Hence we should select the longest straight line segment to compute the depth of object to minimize the error.

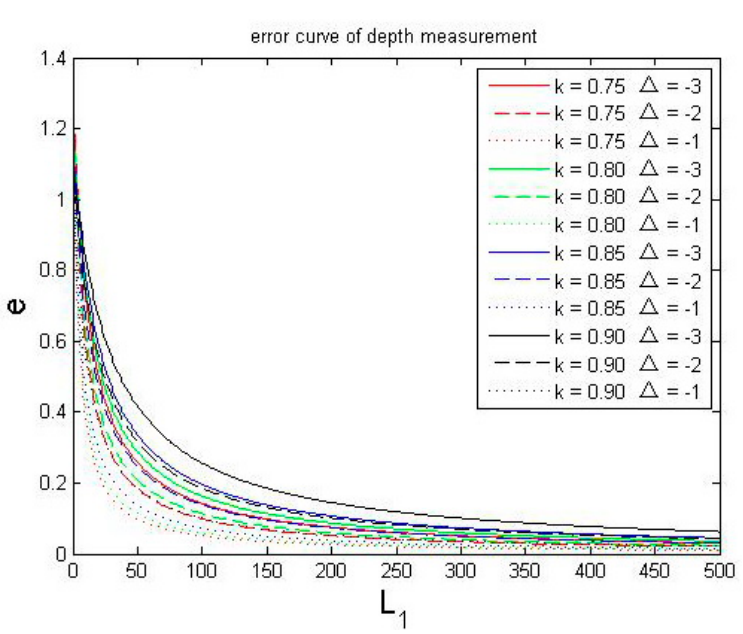

(a)

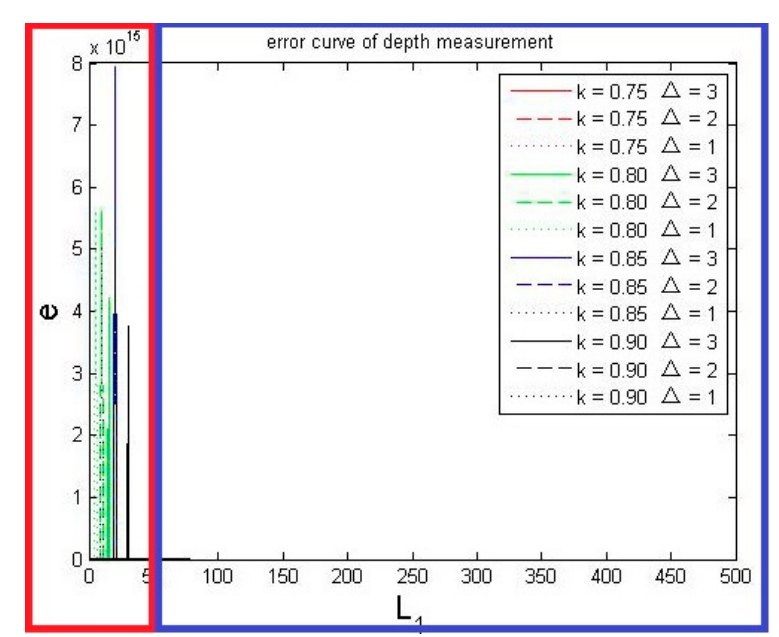

(b) 


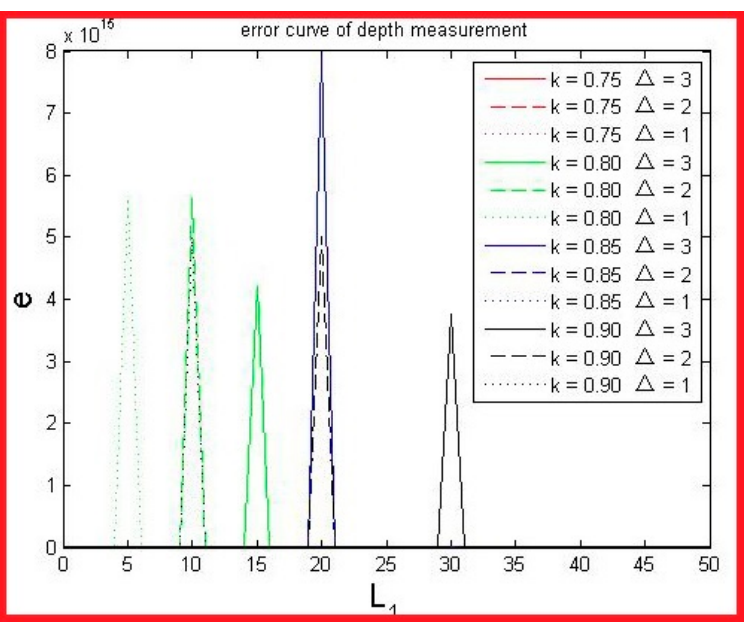

(c)

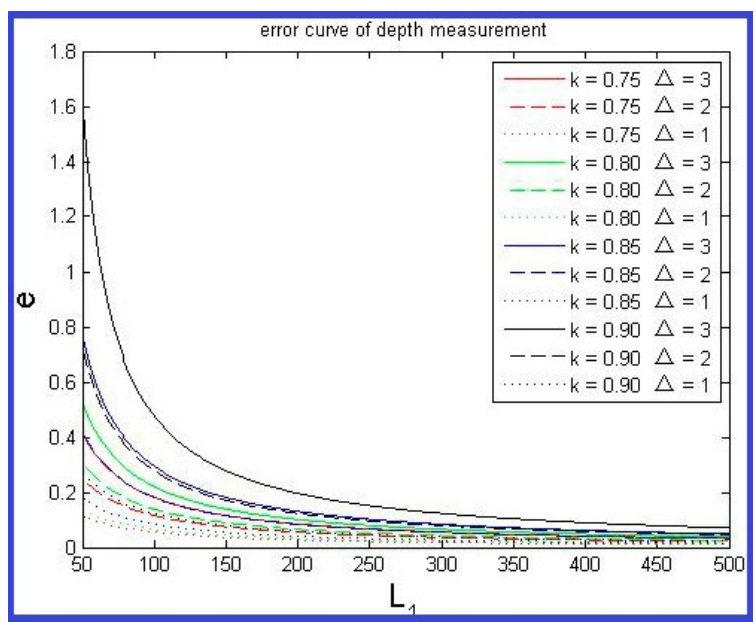

(d)

Figure 3. Relation of depth measurement error to the length of straight line segment L1. (a) Error curve when $\Delta \leqslant 0 ; \quad$ (b) Error curve when $\Delta>0 ; \quad$ (c) Error curve when $L_{1} \in[0,50]$ and $\Delta>0$, namely this's a left part of Figure (b); (d) Error curve when $L_{1} \in[50,500]$ and $\Delta>0$, namely this's a right part of Figure (b).

It also is proved by experiment method that the length of $L_{1}$ is longer the error is smaller. In the experiment, we select the longest straight line segment, the shortest one, the middle length one, and the random one to compute the depth of object, respectively. 103 pairs of images (resolution $1280 \times$ 1024) were used, and there were the depth of 309 objects in the images to be measured. Tab 1 shows the experiment result of the above 4 classes straight line segment selected to measure depth. For every class straight line segment, different object have different length, so value range of the length of every class line segment is showed in the Tab 1. It indicates that we should select the longest straight line segment to compute the depth of object.

Tab 1. Relation of depth measurement error to the 4 classes straight line segment (length unit: pixel)

\begin{tabular}{lllll}
\hline & $\begin{array}{l}\text { The method } \\
\text { of the longest } \\
\text { line }\end{array}$ & $\begin{array}{l}\text { The method of } \\
\text { the shortest } \\
\text { line }\end{array}$ & $\begin{array}{l}\text { The method } \\
\text { of the } \\
\text { middle line }\end{array}$ & $\begin{array}{l}\text { The method } \\
\text { of the } \\
\text { random line }\end{array}$ \\
\hline $\begin{array}{l}\text { Length of the shortest line } \\
\begin{array}{l}\text { Length of the longest line } \\
\text { Average length }\end{array}\end{array}$ & 481.19 & 0.15 & 21.29 & 2.31 \\
$\begin{array}{l}\text { Average error of } \\
\text { measurement }\end{array}$ & 205.71 & 22.81 & 362.41 & 377.35 \\
\hline
\end{tabular}

Different object have different length of the longest straight line segment which consist of the 2 SIFT points on the object. We counted the longest ones on the 309 objects and got the piecewise relations of depth measurement error to the length of straight line segment, and show them into a diagram (Figure 4). It illustrates that the longer straight line segment the smaller error, but the change of percentage error is very little when the length is greater than a certain number (e.g. 200). The experiment result coincides with the theoretical analysis of percentage error (Figure 3 ). 


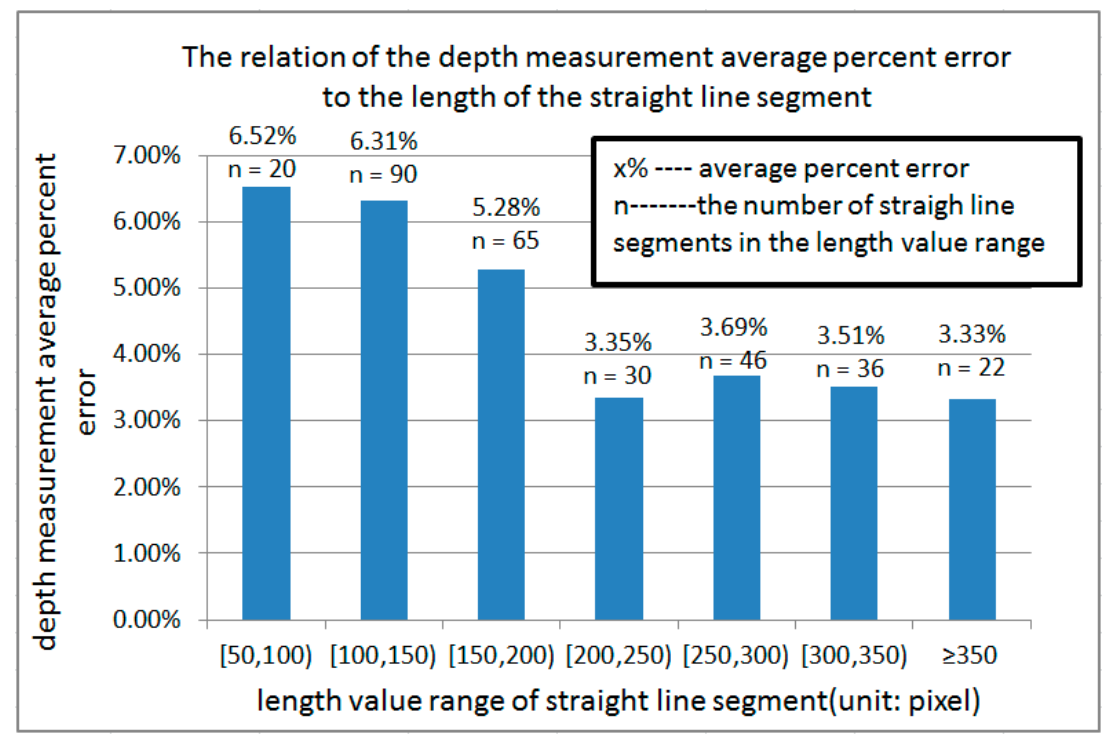

Figure 4. Experiment result of the relation of the depth measurement average percent error to the length of the straight line segment.

\subsection{Decrease Time Complexity}

In a 2-D plane, $n$ points can form $C(n, 2)$ straight line segments. The time complexity is $O\left(n^{2}\right)$ if we compare a line segment length with others one by one to select the longest one. The amount of computation will increase sharply when the value of $n$ is very large. Therefore, we must design an algorithm to cut down the computing time and find the longest line segment rapidly. The convex hull theory is employed to solve this problem.

If we assume that $\mathrm{CH}$ is a convex hull of a given points set $Q, \mathrm{CH}$ is defined as the unique minimal convex set containing $Q$. it implies all of the points in set $Q$ must be in or on the boundary of convex hull $\mathrm{CH}$. Therefore, If there are $n$ points in the $Q$, then we can get $C(n, 2)$ straight line segments which consist of the all points. The two endpoints of the longest one must be on $\mathrm{CH}$.

Convex hull of points set $Q$ can be got by the method of Graham scan. The time complexity of the method is $O(n \lg n)$. We assume that there are $n$ points in the set $Q$, and $m$ points among $n$ belong to the convex hull of set $Q$. Thus we could find the longest straight line segment from the $C(m, 2)$ straight line segments instead of $C(n, 2)$ ones. In general, $m \ll n$ when the $n$ is a large number. It means we can decrease the computation complexity of seeking the longest straight line segment by using the convex hull.

\subsection{Algorithm for selecting a pair of straight line segments}

As similarity measurement is employed to match SIFT feature points $[23,24]$, the wrong matching points exist, although it is very few. If only one of the endpoints of the pair of straight line segment that were used to compute the depth of object was a wrong matching, the value of depth measurement would be widely inaccurate. Hence, we should select the longest straight line segment whose two endpoints all must be matched the corresponding SIFT feature points in image $B$, correctly.

Because, respectively, the image $A$ and $B$ is captured when the object distance is $u$ and $u+d$ under the condition of keeping the camera intrinsic unchanged, we can use similarity of polygon that consist of the SIFT matching points on the image $A$ and image $B$ to tell the matching point is wrong or not. In our experiment the polygon is triangle. In other words, if the triangle $\triangle A B C$ that 
consists of 3 SIFT feature points in image $A$ is similar to the corresponding one $\Delta A^{\prime} B^{\prime} C^{\prime}$ in image $B$, then there are no wrong matching points in the three vertices. Otherwise, there is one wrong matching point at least.

The differences between every corresponding angle of the two triangles are employed to decide whether the two triangles are similar, and we call the maximal angle difference is Angmax. If $A n g_{\max }<T_{a}$, then the two triangles are similar. Where the $T_{a}$ is a threshold.

The similarity triangle one of whose side is the longest line segment should be find out, only in this way we can avoid the wrong endpoints and minimize the error of depth measurement at the same time. It is impossible to determine which one or more vertices of triangle is the wrong matching point(s), when the triangle $\triangle A B C$ is not similar to $\triangle A^{\prime} B^{\prime} C^{\prime}$. Therefore, an algorithm for selecting a pair of straight line segments which are used to compute the depth is proposed. The main idea in the algorithm is as the following: we assume that the number of the triangles one of whose side is the longest straight line segment $L$ is $n_{1}$ in image $A$, obviously, there are $n_{1}$ corresponding triangles in image $B$, but only $n_{2}$ pairs of triangle is similar. The $L$ can be used to compute depth of object if $n_{2} / n_{1}>T_{s}$, where $T_{s}$ is a threshold. Otherwise, we should decide whether the next longest straight line segment is the one that we are seeking by the above method. The algorithm of selecting a pair of straight line segments is shown in the Tab 2.

Tab 2. The algorithm of selecting a pair of straight line segments

Input: $A$ sub-region in image $A$ and its matched one in image $B$ of the same object; The points set $Q$ consist of the SIFT feature points of the object in image $A$, and in image $B$, the points set $Q^{\prime}$ consist of the matched points of $Q$.

Output: A pair of straight line segment used to compute depth of object.

Step1: Assume $i=1$.

Step2: Compute to obtain the points set $P$, and the $P$ is the convex hull of $Q$.

Step3: Compute to obtain the length of all of the straight line segment which is composed of the any 2 points in $P$, and put them in their length order, from the longest to the shortest.

Step4: Count $n_{1}$ and $n_{2}$, where $n_{1}$ is the number of the triangles one of whose side is the $i$-th long line segment in image $A$, and $n_{2}$ is the number of the corresponding and similar triangles in image $B$.

Step5: If $n_{2} / n_{1}>T_{s}$, go to step6; else, $i=i+1$, and go to step4.

Step6: The $i$-th long straight line segment in image $A$ and the corresponding one in image $B$ are the pair of straight line segments, which will be used to compute the depth of object.

The method 1 is to compute the depth after the wrong matching points have been deleted by the above algorithm. The method 2 is to compute the depth by the longest straight line segment directly. The measurement errors of the two kinds of methods are showed in Tab 3. There are 3 objects whose depth should be computed in the given images, and it is showed in Figure 6. We called the 3 objects obj1, obj2, obj3, respectively. In method 2, the error percentage of depth measurement of obj2 is $22.91 \%$, because 1 or 2 points of the two endpoint of the longest straight line segment are matched wrong. It is a very big error. But, in method 1, the error is decreased sharply for the $3^{\text {rd }}$ long straight line segment was used to compute the depth of object by the above algorithm. Although its length is not the longest, it is the best choice to compute the depth information. 
Tab 3. Measure result comparison of the two kinds of methods (unit: distance-mm; length of line segment - pixel; angle-degree. $D=600 \mathrm{~mm}, T_{a}=3^{\circ}$ )

\begin{tabular}{|c|c|c|c|c|c|c|c|c|c|c|c|}
\hline \multirow[b]{2}{*}{ object } & \multirow[b]{2}{*}{$\mathrm{t} \quad \mathrm{GT}^{* 1}$} & \multicolumn{6}{|c|}{ Method 1} & \multicolumn{4}{|c|}{ Method 2} \\
\hline & & Ang $_{\max }$ & $i-\mathbf{t h}^{*_{2}}$ & ${ }^{2} \quad \mathrm{~L}_{1}$ & $\mathbf{L}_{2}$ & $\mathrm{MD}^{* 3}$ & $\mathrm{EP}^{* 4}$ & $L_{\max 1}$ & $L_{\max 2}$ & $\mathrm{MD}^{* 3}$ & $\mathbf{E P}^{* 4}$ \\
\hline obj1 & 3565.38 & 0.35 & 1 & 269.00 & 229.34 & 3470.07 & $2.67 \%$ & 269.00 & 229.34 & 3470.07 & $2.67 \%$ \\
\hline obj2 & 3389.00 & 2.62 & 3 & 141.41 & 121.31 & 3620.50 & $6.83 \%$ & 190.09 & 154.59 & 2612.66 & $22.91 \%$ \\
\hline obj3 3 & 3758.01 & 0.55 & 1 & 302.35 & 257.87 & 3477.98 & $7.45 \%$ & 302.35 & 257.87 & 3477.98 & $7.45 \%$ \\
\hline
\end{tabular}

${ }^{* 1}$ GT is the ground truth of the depth of object.

${ }^{*}{ }_{2} \quad$ The $i$-th means that the $i$-th long straight line segment was used to compute the depth of object.

$*_{3}$ MD is measured depth.

${ }^{*} \quad \mathrm{EP}$ is error percentage.

\section{Experiments}

\subsection{Images Acquirement}

To estimate the depth of objects in image, we must acquire two images (image $A$ and image $B$ ). Figure 5 shows the process. Firstly, the image $A$ is acquired at the place where the distance between the object and the camera is $u$. Next, the camera intrinsic is remain unchanged and the camera is moved along the optical axis to the place where the object distance is $u+d$, and the image $B$ is acquired. To obtain the actual distance of the objects, the objects to be measured were sitting at the specified locations. In Figure 5(a), the values of $x_{1}, x_{2}, y_{1}, y_{2}, d$ and $u$ can be got by using the manual measure, then we can depend on the values and the geometry and size of the objects to compute the actual depth of objects. For example, in Figure 5(a), the object obj1 is a cylinder. Assume its diameter is $D$, then the actual depth of obj1 is $\left(\left(u+y_{2}+D / 4\right)^{2}+x_{1}{ }^{2}\right)^{1 / 2}$. Similarly, the actual depth of obj2 and obj3 are $u$ and $\left(\left(u+y_{1}+y_{2}\right)^{2}+x_{2}^{2}\right)^{1 / 2}$, respectively.

Ordinarily, it is very difficult that the camera is moved along the optical axis rigorously. In practical operation, the camera can be installed on a horizontal guide rail or platform on which a straight line have been drawn, then the camera is moved along the guide rail or the straight line on the platform. It makes the moving direction coincident with the optical axis approximately. In our experiments, the camera is put on a horizontal platform which consists of several identical lab benches and moved along a straight line which had drawn on the platform beforehand. The scene is showed in Figure 5(b). 


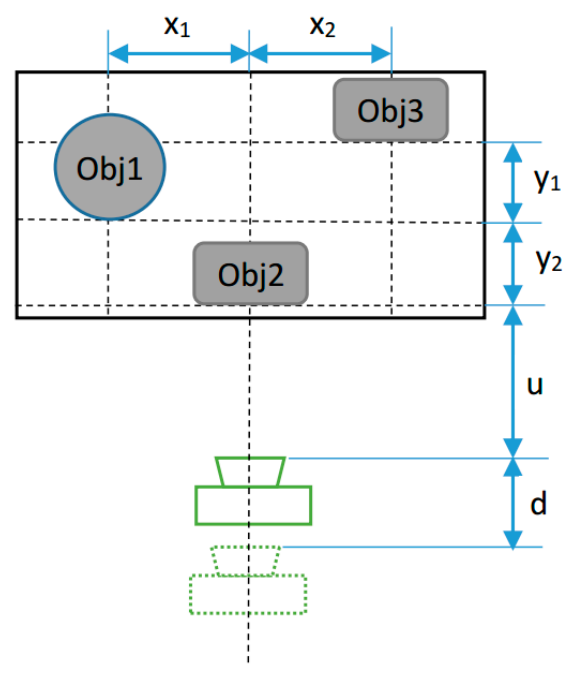

(a)

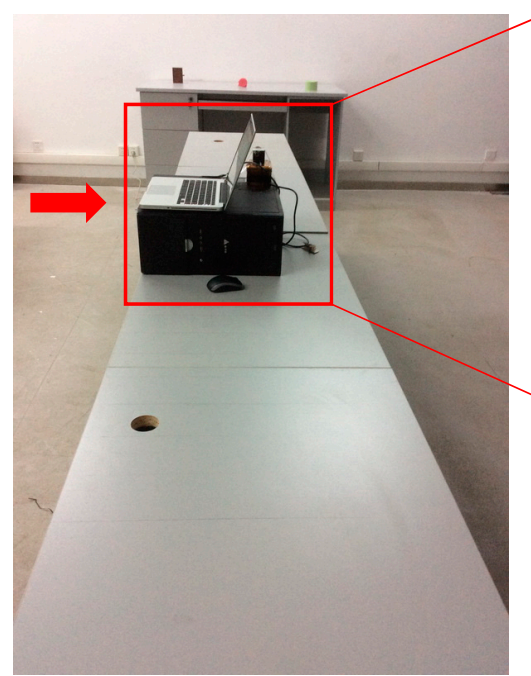

(b)

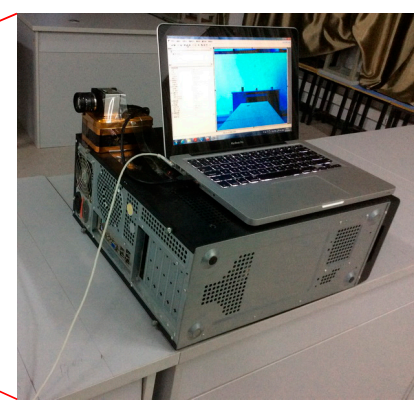

Figure 5. (a) is the schematic diagram of the process acquiring the two images; (b) is the real scene which was used to capture the image $A$ and $B$

\subsection{Experiments Procedure}

To test and verify our approach on depth measurement is effective, we acquire the images and follow the method steps to carry out experiments. Figure 6 illustrates the procedure of our approach. There are original images, the depth image of objects and some significant interim result images, etc. in Figure 6.

From the Figure $6(\mathrm{f})$, we can see that a whole image of measured object is not necessary to measure the depth of object. Because the depth can be compute by the lengths of a pair of straight line segments. And the pair of straight line segments can be got by a part of the image of object, usually. Consequently, our method is robust to occlusion or partial loss of image of object.

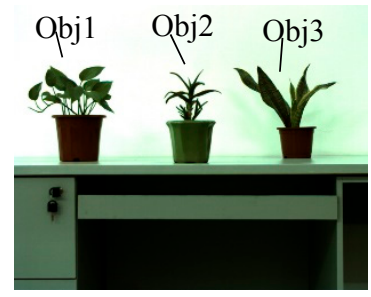

(a)

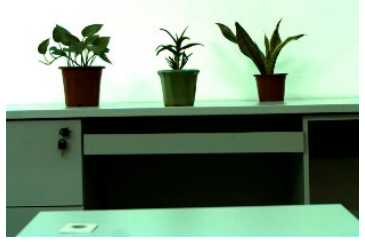

(b)

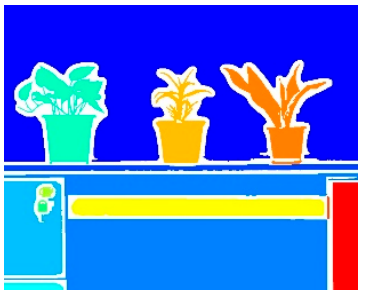

(c)

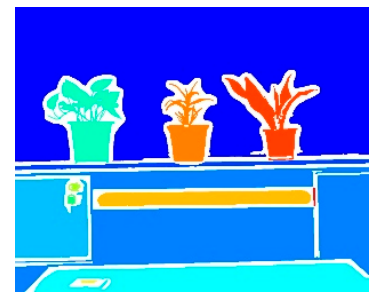

(d)

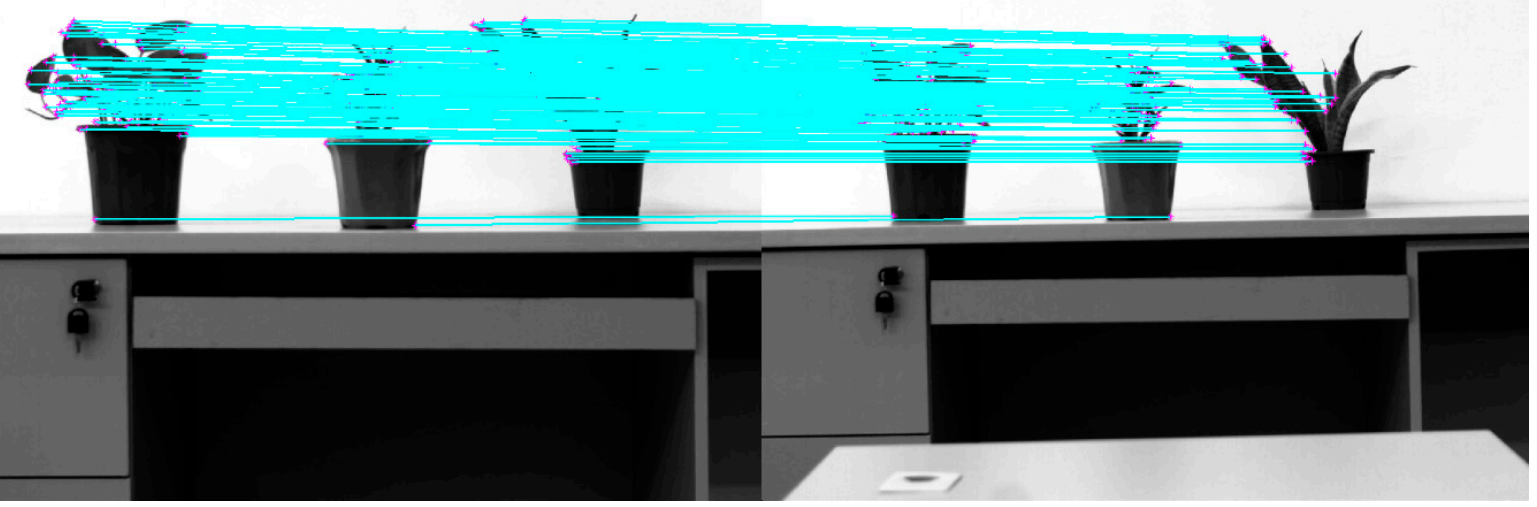


(e)

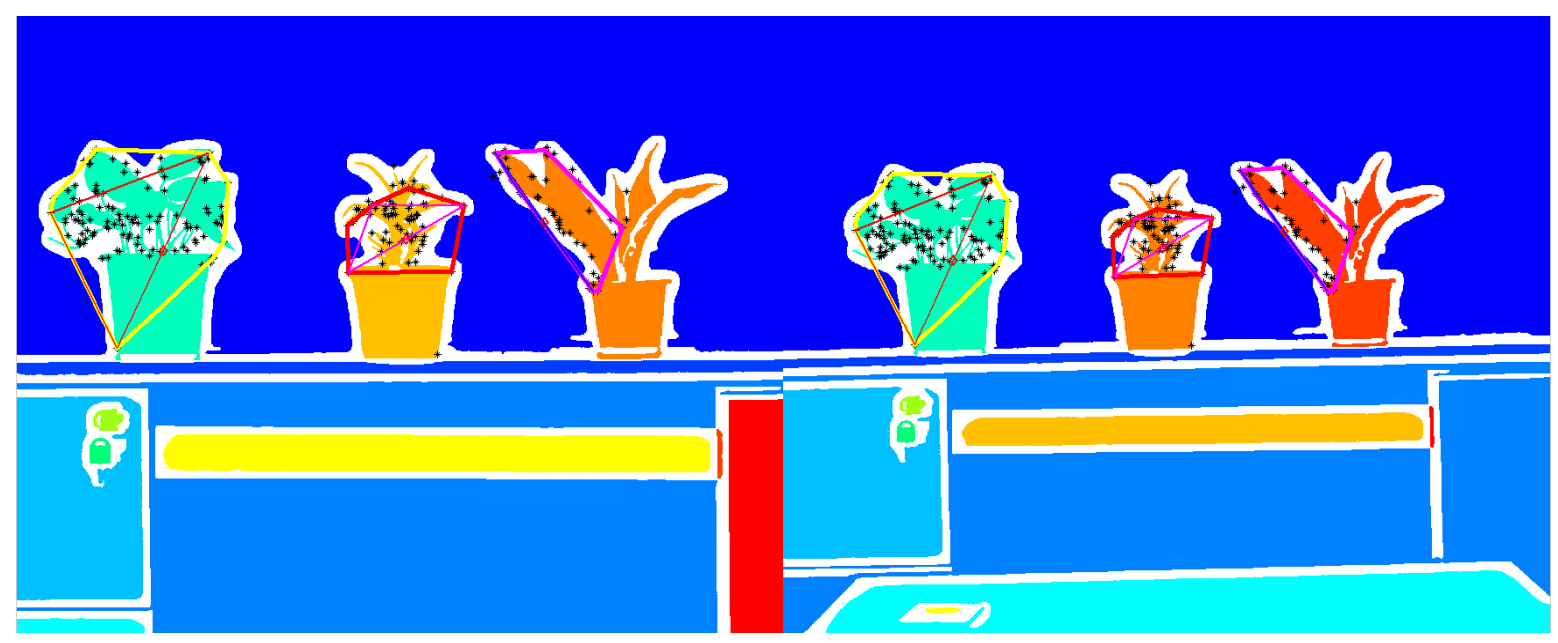

(f)

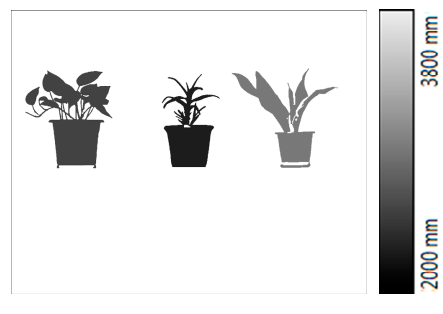

(g)

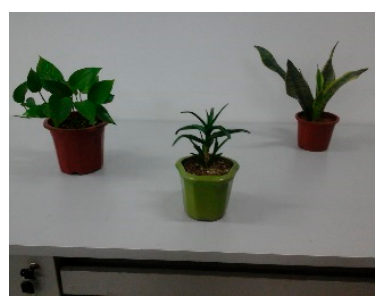

(h)

Figure 6. The experimental procedure. (a) The original image $A$ was acquired at the place where the object distance is $u$. (b) The original image $B$ was acquired at the place where the object distance is $u+d$. (c) and (d) are the segment results of the image (a) and (b), respectively. In order to make the sub-regions clear, we label the different sub-region with different color. (e) SIFT feature points detected and matched. The true-color image $A$ and $B$ are converted to grayscale, then, detect the SIFT feature points in them, and match the points. The locations of pink star "*" are the locations of SIFT feature point. (f) Matching the objects by taking advantage of the SIFT feature points and the segment results. Then we get the convex hull of the set of SIFT feature points of each object to be measured by the method of Graham scan. Lastly, the pair of straight line segments are chose by the algorithm descripted in section 4.3. They are labeled by drawing a sign " $\diamond$ " on it. (g) The depths of objects are expressed by the gray-scale. The gray value of pixel is smaller, the value of depth is smaller too. (h) This image shows the spatial relationship of the objects. Namely, there are the different distances between camera and the different measured objects. It is convenient for us to compare with image (g).

\subsection{Our Approach Compares with Others'}

We compare our experiment result with Bumblebee's, Zhou's[17] and Fang's[20], as shown in Figure 7. There are three scenes, and different target objects measured are contained in each scene. Figure 7(a) and (b) are the images $A$ and $B$ which are captured at different object distance by a common camera, respectively. The spatial relationships of the objects measured are shown in Figure 7(c). The images are all taken from a top down view. They are convenient for us to estimate a target object is near or far to the camera, roughly.

Figure 7(d),(e),(f),(g) are the depth images which measured by Zhou's method[17], Bumblebee device, Fang's method[20] and our method, respectively. In Figure 7(d), Zhou gets dense depth maps, but they are relative depth information instead of absolute depth. Only depth of partial scene are measured in Figure 7(e),(g), but they are absolute depth, namely they are object distance values 
which are represented by different pixel values. Both the gray pixels in Figure 7(e) and the white pixels in Figure $7(\mathrm{~g})$ indicate that the depth information of the place could not be measured. In Figure $7(\mathrm{e}),(\mathrm{f}),(\mathrm{g})$, the different color or gray pixels mean different depth value.

There are some obvious wrong depth information in Figure 7(d). For example, in every image, the gray levels of the two objects which are pointed by red arrows are similar. It means that their depths that were measured by the Zhou's method are similar, but, at fact, the disparity of the depth values is very big. The reason for this error is that the Zhou's method can only measure the defocus degree, but it can't judge the focal plane in front of or behind the imaging plane. Furthermore, the depth of the object which is pointed by blue arrows should be as same as its surroundings. But the results of measurement are not consistent with the ground true. Because the texture of the image affects the construction accuracy of dense depth map, which are obtained by applying matting Laplacian to perform sparse depth map interpolation[17].

We also can see that there are several obvious errors of depth measurement in Figure 7(e), as the white arrows point. At fact, the depth values of the two places are very different, but the depth values measured by Bumblebee device are similar. So the colors of the two places are very similar in the images. In addition, the outlines of the objects in the depth images are different from the real objects. It was caused by the error of measurement.

Figure 7(f) is result of depth image by Fang's method. The method is faster than state-of-the-art method, and it can achieve quasi real-time performance. But the accuracy of depth information is not good. It is difficult for us to find the outlines of the objects from the depth image, especially to the outlines of the small objects. In addition, the method needs a dataset to train and learn.

Figure $7(\mathrm{~g})$ is result of depth image by our method. We can see that we can obtain a high accuracy absolute depth of the target objects and the outlines of the objects are clear and accurate in the depth images by our method. In Figure 7(g), only the depth of watering pot head is showed, but the depth of the watering pot body cannot be measured. Because the watering pot was divided into two parts - the watering pot head and the watering pot body after image segmentation, and no enough number of SIFT feature points could be detected on the watering pot body for the color of the body is very close to the background. Usually, if no less than 4 SIFT points are detected on an object, and then we can use them to compute the depth of objects. Therefore, our method works well in most cases.

(a)
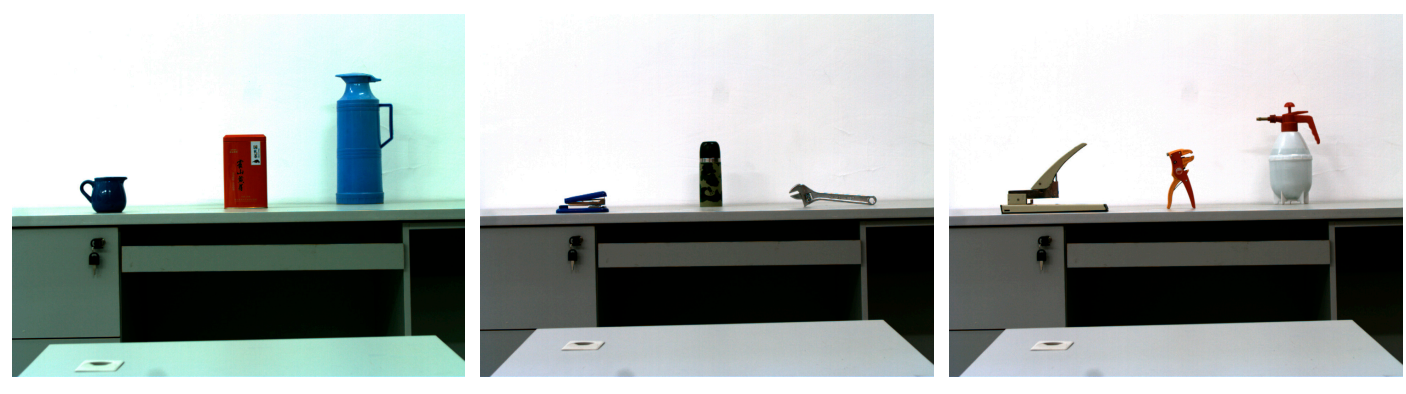

(b)
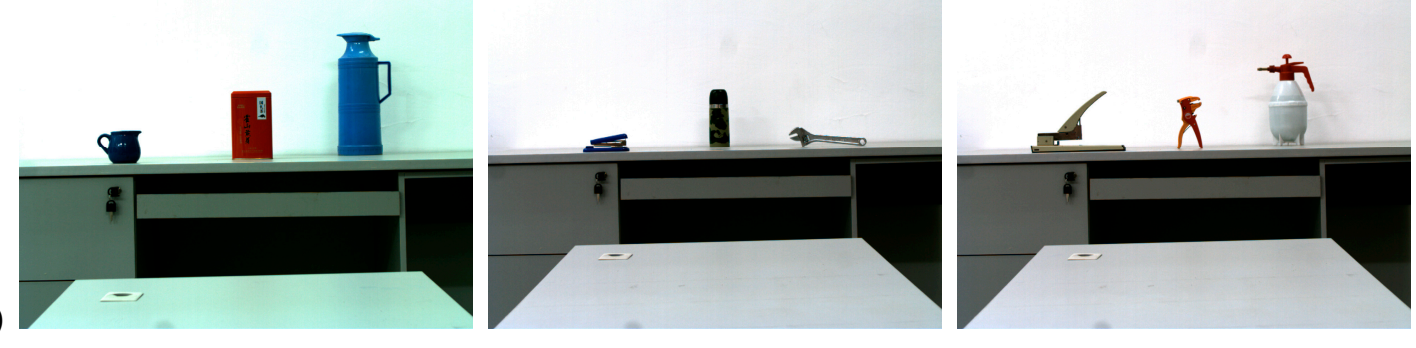
(c)
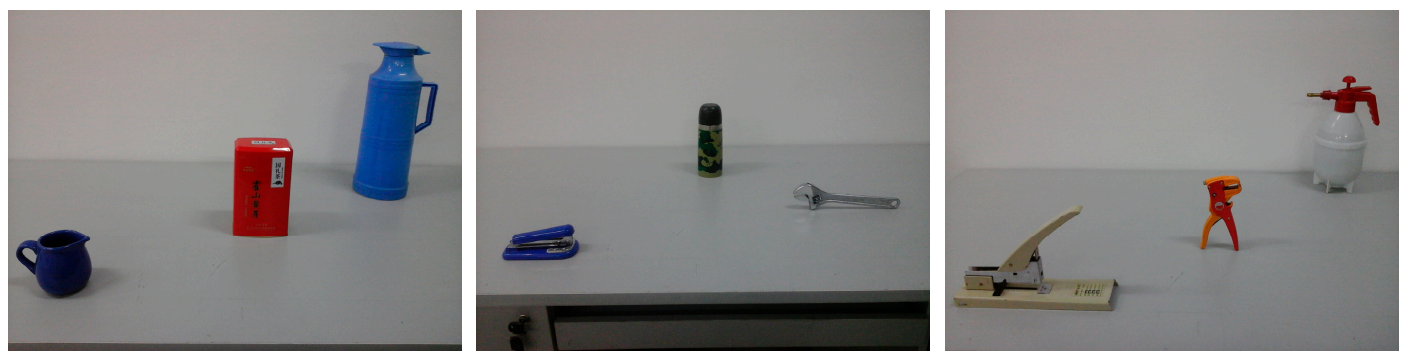

(d)
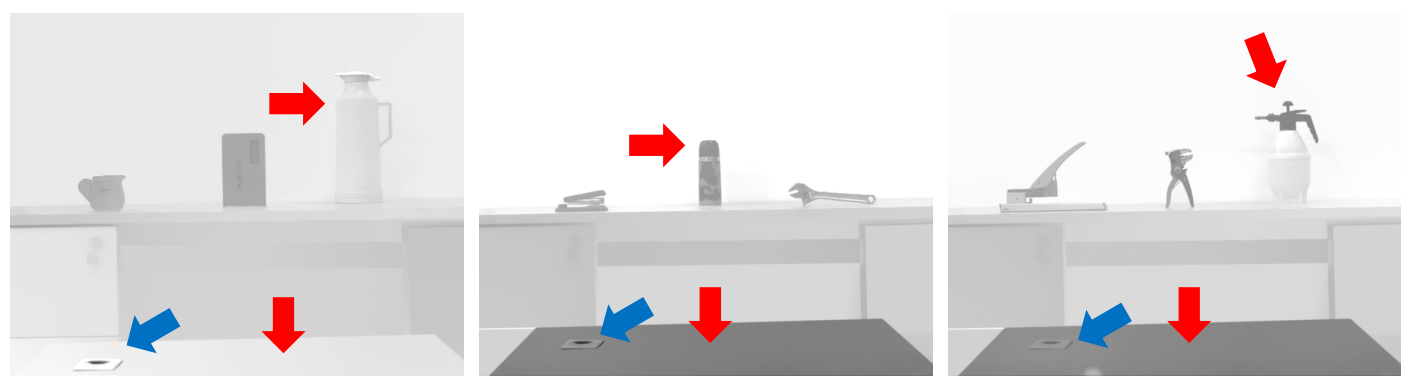

(e)
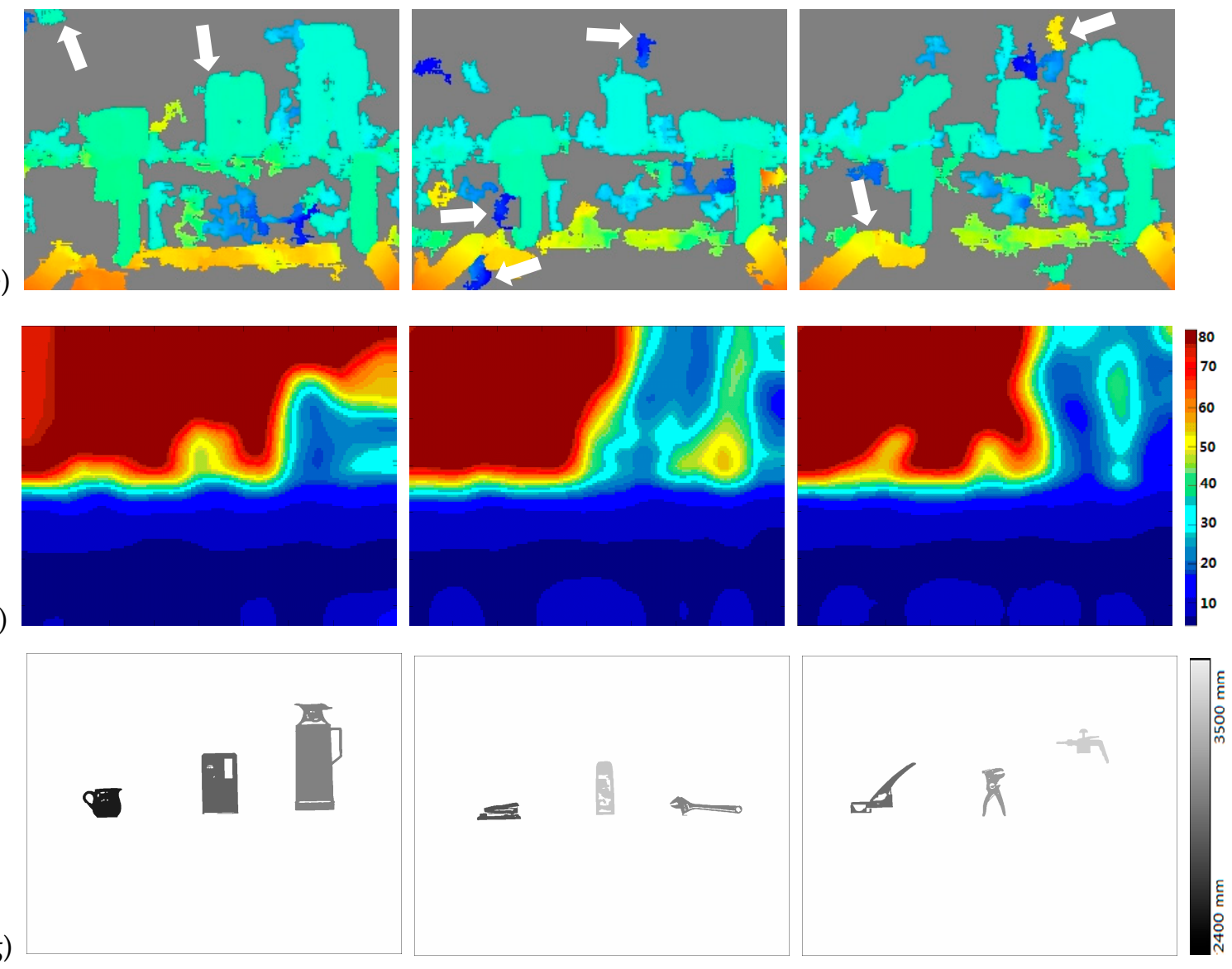

Figure 7. Comparison of depth estimation result of different approach. (a)This row is original images $A$ taken by a common camera; (b) This row is original images $B$ taken by the same camera, only the object distance is different;(c) This row images, which are taken from a top down view, show the space relationship of measured objects. They are convenient for us to judge an object is near or far to the camera, roughly; (d) This row is the depth images that measured by Bumblebee device; (e) This row is the depth maps that measured by the method of Zhou[17]; (f) This row is the depth maps that measured by the method of Fang[20]. The source code is available on GitHub (https:/github.com/king9014/rf-depth); (g) This row is the depth images that measured by our method. 
Experiment images of the other ten scenes are showed in Figure 8. From left to right, each column is image $A$, image $B$, results of our method, results of the method of Zhou, and the rough spatial relationship of the measured objects, respectively.

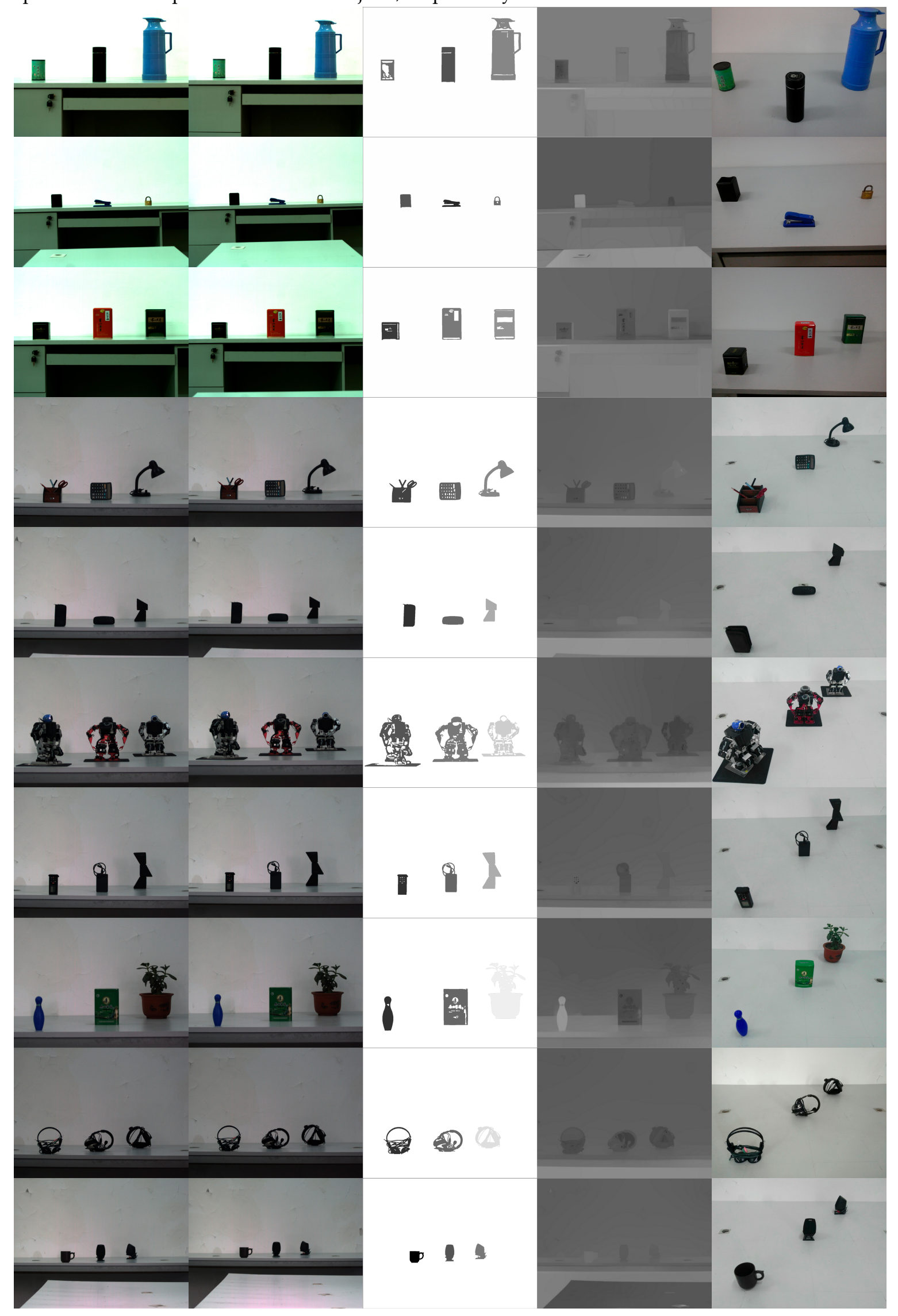


Figure 8. The ten scenes experiment images. The first column is original images $A$. The second column is original images $B$. The third column is the depth images that measured by our method. The forth column is the depth images that measured by the method of Zhou. The last column images, which are taken from a top down view, show the space relationship of measured objects. They are convenient for us to judge an object is near or far to the camera, roughly.

In order to determine the degree of accuracy of result of our method, we took 191 groups images for experiments. There are 382 images, for a group includes 2 images. And there are 3 target objects measured in a group, so 573 depths of objects should be measured. In our implementation, we use the same parameters for the whole experimental, i.e. $T_{p}=0.6, T_{s}=0.6, T_{\theta}=3^{\circ}$. We compare the result of our method with the result of Kouskouridas et al's method[19], as shown in Tab 4.

Tab 4. Comparison between our method with Kouskouridas et al's method

\begin{tabular}{llc}
\hline & Our method & Kouskouridas et al's method \\
\hline Device required & camera & camera and laser depth measurement device \\
Number of images required & 2 & $\geqslant 5$ images for every measured object \\
Is a sample database required? & NO & YES \\
Can the depth of object which does not & & NO \\
registered in the database be measured? & YES & $9.89 \%$ \\
Average error percentage & $5.14 \%$ & \\
\hline
\end{tabular}

In the Kouskouridas' method, the SIFT are employed to obtain the depth of objects, too. Firstly, a database should be built and the measured objects must be registered in the database, or their depth cannot be measured. The database comprise object distance, a lot of images and their SIFT. The images of each measured object are taken from different object distance and different angle of view. The uniform and simple background is required in the images. The object distance is obtained by a laser device. Then, the data in the database is used for training of their algorithm. For an image, the depth of the objects in the image can be computed only when the objects had been stored in the database. Above all, the SIFT feature points should be detected. Then, the center of mass of feature points set and the average distance $d_{m}$ between it and every feature point should be calculated. Lastly, the depth of objects can be computed by using $d_{m}$ and some data in the database.

Tab 4 shows advantages of our method are less devices, easy operation, no sample database, and less measurement error.

Figure 9 is the comparison of depth of the objects measured by our method with the ground truth. The horizontal axis represents the serial number of the measured objects. The vertical axis represents the depth of the measured objects. The red curve is the depth measured by our method, and the blue one is the ground truth, and. The maximum error is $16.1 \%$, and the minimum error is $0.01 \%$, and the average error is $5.14 \%$. Figure 9 indicate that our method is effective. 


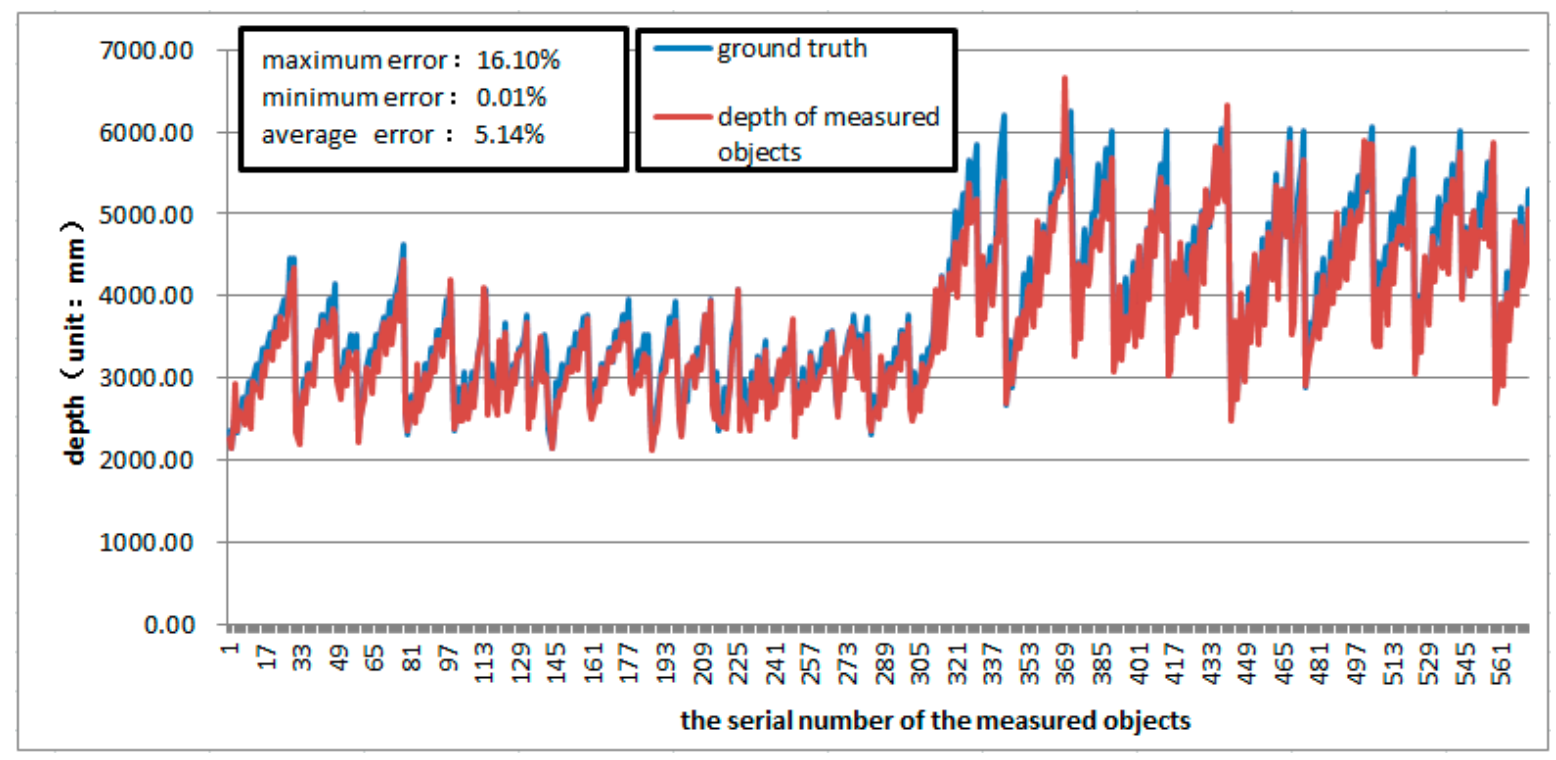

Figure 9. Comparison the depth of the objects measured by our method with the ground truth.

\section{Discussions}

Firstly, image segmentation is employed in our approach. The accuracy of depth information of measured objects may be influenced by the result of image segmentation. It is not easy to separate the different types of objects measured from an image with complex background using the same parameter. The depth accuracy would be reduced greatly if the result of segmentation is wrong. Therefore, how to extract the depth information of the target objects with complex background is one of our next works.

Secondly, our algorithm is a time consuming. Because the image segmentation, SIFT feature points detecting and matching are employed in our algorithm. The configuration of the computer which used to the experiment is as follow: memory 16G, CPU i7-4810MQ, 2.80GHz, 4 cores. The codes were run in matlab2012b. It takes about 139.6 seconds time to extract the depth information from an image (resolution $1280 \times 1024$ ) by our method. Among of them, about 95 seconds are used for the image segmentation, and about 43.5 seconds are used for the SIFT feature points detecting and feature points matching, and about 1.1 seconds are used for the rest. Therefore, we will focus on how to reduce the computational complexity in our next research.

Lastly, in our method, the images of the same object in the images $A$ and $B$ are matched by using SIFT feature points, and a pair of straight line segments which are used to compute the depth of object are chose according to the algorithm descripted in section 4.3. When the length of the straight line segment is less than 150 pixels, the shorter the length of straight line segment is, the lower the accuracy of computed depth is. Thus, our method has a low accuracy to the very small objects. In addition, the depth cannot be measured unless there are no less than 4 SIFT feature points being detected on the target objects. Therefore, our next research will focus on how to improve the depth measurement accuracy of small objects and how to measure the depth of target object whose number of SIFT feature points is less than 4. 


\section{Conclusions}

A novel method on depth measurement is proposed in this paper, according to our analysis on relative work of the other researchers and their shortcoming. Firstly, in our method, the required device is very economic and it is convenient to operate. Only two images of the same scene should be provided in our method. The two images are captured by a camera. The camera is a common one and its price is cheap usually. The camera needs not to be calibrated and no parameter is needed to be adjusted during imaging. Secondly, our method gets the absolute depth of object instead of the relative depth; and it is robust to occlusion or partial loss of object, because the depth can be compute by the lengths of a pair of straight line segments, and the pair of straight line segments can be got by a part of the image of object, usually. Lastly, the superiority of our method has been shown through the comparisons with the methods of references[17,19,20] and the method of Bumblebee. The effectiveness and practicability of our method is proved by our experimental results.

Acknowledgments: This research work is supported by the grant from the National Natural Science Foundation of China, Nos. 91120307, 91320301, 61304122, and 61672204, the grant from the Scientific Research Foundation of Education Department of Anhui Province, Nos. KJ2017A541, KJ2015A162, KJ2013B230, KJ2013A226, the grant from the Key Constructive Discipline Project of Hefei University, No. 2016xk05, the grant from the Quality Engineering of Higher Education of AnHui Province, Nos. 2015ckjh047, 2015ckjh048, 2015ckjh058, 2015ckjh061, 2015zy054, 2015zjjh026, and 2015zdjy141, the grant from Outstanding Youth Talent Foundation of Hefei University, No.16YQ06RC, the grant from the Scientific Research Foundation of Hefei University, No. 16ZR14ZDA, the grant from the Education Research Foundation of Hefei University, No. 2016mkjy04.

Author Contributions: all authors of the paper have made significant contributions to this work. Lixin He conceived the idea of work, wrote the manuscript, and led the project. Jing Yang assisted to conceived the idea of work and analyzed the experiment data. Bing Kong analyzed the experiment result and give advice about writing. Can Wang collected the original data of experiment and participated in programming.

Conflicts of Interest: All authors of the paper declare no conflict of interest.

\section{References}

1. Santos, D. G.; Fernandes, B. J. HAGR-D: A Novel Approach for Gesture Recognition with Depth Maps. Sensors. 2015,15,28646-28664.

2. Huang, H. C.; Hsieh, C. T. An Indoor Obstacle Detection System Using Depth Information and Region Growth. Sensors. 2015,15,27116-27141.

3. Hosni, A.; Rhemann, C. Fast Cost-Volume Filtering for Visual Correspondence and Beyond. IEEE Trans. Pattern Anal. Mach. Intell. 2013, 35, 504-511.

4. Jiang, H.; Xiao, J. X. A Linear Approach to Matching Cuboids in RGBD Images. IEEE Conference on Computer Vision and Pattern Recognition (CVPR)2013. 2171-2178.

5. De-Maeztu, L.; Mattoccia, S. Linear stereo matching. IEEE International Conference on Computer Vision (ICCV)2011. 1708-1715.

6. Tao, M. W.; Hadap, S. Depth from Combining Defocus and Correspondence Using Light-Field Cameras. IEEE International Conference on Computer Vision (ICCV)2013. 673-680.

7. Chen, C.; Lin, H. T. Light Field Stereo Matching Using Bilateral Statistics of Surface Cameras. IEEE Conference on Computer Vision and Pattern Recognition (CVPR)2014. 1518-1525. 
8. Tao, M. W.; Wang, T. C. Depth Estimation for Glossy Surfaces with Light-Field Cameras. European Conference on Computer Vision( ECCV )2014. Workshops2015. 533-547.

9. Tao, M. W.; Su, J. C. Depth Estimation and Specular Removal for Glossy Surfaces Using Point and Line Consistency with Light-Field Cameras. IEEE Trans. Pattern Anal. Mach. Intell. 2016, 38, 1155-1169.

10. Wang, T. C.; Efros, A. A. Depth Estimation with Occlusion Modeling Using Light-Field Cameras. IEEE Trans. Pattern Anal. Mach. Intell. 2016, 38, 2170-2181.

11. Nayar, S. K.; Nakagawa, Y. Shape from Focus. IEEE Trans. Pattern Anal. Mach. Intell. 1994, 16, 824-831.

12. Subbarao, M.; Tyan, J. K. Selecting the optimal focus measure for autofocusing and depth-from-focus. IEEE Trans. Pattern Anal. Mach. Intell. 1998, 20, 864-870.

13. Pentland, A. P. A New Sense for Depth of Field. IEEE Trans. Pattern Anal. Mach. Intell. 1987, 9, 523-531.

14. Subbarao, M.; Gurumoorthy, N. Depth recovery from blurred edges. IEEE Conference on Computer Vision and Pattern Recognition(CVPR), Jun1988. 498-503.

15. Rajagopalan, A. N.; Chaudhuri, S. Depth estimation and image restoration using defocused stereo pairs. IEEE Trans. Pattern Anal. Mach. Intell. 2004,26,1521-1525.

16. Favaro, P.; Soatto, S. Shape from defocus via diffusion. IEEE Trans. Pattern Anal. Mach. Intell. 2008, 30, 518-531.

17. Zhuo, S. J.; Sim, T. Defocus map estimation from a single image. Pattern Recogn. 2011, 44, 1852-1858.

18. Zhou, C.; Nayar, S. What are good apertures for defocus deblurring? IEEE International Conference on Computational Photography (ICCP)2009. 1-8.

19. Kouskouridas, R.; Gasteratos, A. Evaluation of two-part algorithms for objects' depth estimation. IET Comput. Vis. 2012, 6, 70-78.

20. Fang, S., Jin, R., Cao, Y. Fast depth estimation from single image using structured forest. IEEE International Conference on Image Processing(ICIP). 2016. 4022-4026.

21. Li, C. M.;Kao, C. Y. Implicit active contours driven by local binary fitting energy. IEEE Conference on Computer Vision and Pattern Recognition(CVPR)2007. 339-345.

22. Li, C. M.;Kao, C. Y. Minimization of region-scalable fitting energy for image segmentation. IEEE Trans. Image Process. 2008,17,1940-1949.

23. Lowe, D. G. Distinctive image features from scale-invariant keypoints. Int. J. Comput. Vis. 2004, 60, 91-110.

24. Lowe, D. G. Object recognition from local scale-invariant features. Proceedings of the Seventh IEEE International Conference on Computer Vision(ICCV),1999. 1150-1157. 